\title{
The electron temperature of the inner halo of the Planetary Nebula NGC 6543
}

\author{
S. Hyung ${ }^{1}$, G. Mellema ${ }^{2}$, S.-J. Lee ${ }^{3}$, and H. Kim ${ }^{4}$ \\ 1 Korea Astronomy Observatory, 61-1 Whaam-dong, Yusong-gu, Taejon 305-348, Korea \\ e-mail: hyung@kao.re.kr \\ 2 Sterrewacht Leiden, PO Box 9513, 2300 RA, Leiden, The Netherlands \\ e-mail: mellema@strw.leidenuniv.nl \\ 3 Dept. of Astronomy and Space Science, Chungnam National University, 220 Kung-dong, Yusong-gu, \\ Taejon 305-764, Korea \\ e-mail: seong@canopus.chungnam.ac.kr \\ 4 Dept. of Earth Science Education, Korea National University of Education, Chung-Buk 363-791, Korea \\ e-mail: vitkim@kao.re.kr
}

Received 14 June 2001 / Accepted 16 August 2001

\begin{abstract}
We investigate the electron temperature of the inner halo and nebular core regions of NGC 6543, using archival Hubble Space Telescope (HST) Wide Field Planetary Camera 2 (WFPC2) images taken through narrow band [O III] filters. Balick et al. (2001) showed that the inner halo consists of a number of spherical shells. We find the temperature of this inner halo to be much higher $(\sim 15000 \mathrm{~K})$ than that of the bright core nebula $(\sim 8500 \mathrm{~K})$. Photo-ionization models indicate that hardening of the UV radiation from the central star cannot be the main source of the higher temperature in the halo region. Using a radiation hydrodynamic simulation, we show that mass loss and velocity variations in the AGB wind can explain the observed shells, as well as the higher electron temperature.
\end{abstract}

Key words. hydrodynamics - stars: AGB and post-AGB - stars: winds, outflows - ISM: kinematics and dynamics - planetary nebulae: individual: NGC 6543

\section{Introduction}

NGC 6543 (PN G096.4+29.9) is one of the more intriguing Planetary Nebulae (PNe) because of its complex morphology. The core nebula (the "Cat's Eye") consists of several shells and rings projected on top of each other. Images taken with the Chandra X-ray telescope have for the first time revealed the central wind-driven bubble (Chu et al. 2001). Along the symmetry axis, the PN shows FLIERs (Fast Low Ionization Emission Regions).

The object is surrounded by a "giant halo" of 50 ', which has an overall spherical morphology, but which at the outer edge consists of many irregularly shaped gas clouds (Middlemass et al. 1989, henceforth MCW89). This giant halo has a low expansion velocity of at most $5 \mathrm{~km} \mathrm{~s}^{-1}$, and despite its chaotic appearance, it does not show any distinct kinematic features (Bryce et al. 1992). One of the brighter filaments is characterized by a high electron temperature of $15000 \mathrm{~K}$, derived from an analysis of the [O III] line ratio (MCW89), but by a much

Send offprint requests to: G. Mellema, e-mail: mellema@strw.leidenuniv.nl lower temperature of $9000 \mathrm{~K}$ when considering the $\mathrm{H} \alpha$ and [N II] $6584 \AA$ A profiles (Meaburn et al. 1991).

Recently, Balick et al. (2001) (henceforth BWH01) analyzed archival Hubble Space Telescope (HST) images and found that the region just outside the bright core shows at least nine circular "rings", which are in fact projected spherical shells. We will refer to this region as the "inner halo" region to distinguish it from the "outer halo" studied by MCW89 and Meaburn et al. (1991). This inner halo looks much more continuous and regular than the outer halo. Rings like the ones seen around NGC 6543 are quite common, and are found around AGB stars like IRC 10216 (Mauron \& Huggins 2000), post-AGB stars like CRL 2688 (Sahai et al. 1998), and other PNe, such as NGC 7027, and $\mathrm{Hb} 5$. The time scale associated with the implied mass loss variations are typically $100-1000$ years, which is too long to associate them with the pulsations of AGB stars, and too short to connect them to thermal pulses. They could originate from variations inherent to the nature of the mass loss mechanism during the AGB phase (Simis et al. 2001), or from stellar magnetic field variations (Soker 2000; Garcia-Segura et al. 2001), or from 
Table 1. WFPC2 archive images.

\begin{tabular}{llll}
\hline \hline Name & Filter & [Ion] line & Exp Time (s) \\
\hline U27Q0103T & F437N & [O III] 4363 & 1200 \\
U27Q010AT & F502N & [O III] 5007 & 600 \\
U27Q010FT & F656N & H $\alpha$ & 200
\end{tabular}

All these observations were performed on September 18, 1994.

the influence of a companion star (Harpaz et al. 1997; Mastrodemos \& Morris 1999).

The central star of NGC 6543 is a Wolf-Rayet star of spectral type [WC 8]. The P Cygni profiles of, for example, $\mathrm{CIV}$ and NIV in its spectrum indicate a terminal wind velocity of $V(\infty)=2150 \mathrm{~km} \mathrm{~s}^{-1}$ (Castor et al. 1981). The chemical abundances in NGC 6543 are likely to be position dependent (Hyung et al. 2000). Its distance is not well known; values derived through statistical methods are around $1 \mathrm{kpc}$, and according to other methods it could be as close as $500 \mathrm{pc}$. We will assume a distance of $1 \mathrm{kpc}$.

In this paper, we investigate the inner halo region by deriving the [O III] 5007/4363 line ratio from archival narrow band HST images, using a proper interstellar extinction correction. This procedure and the results are described in Sect. 2. In Sect. 3 we attempt to explain the results using both photo-ionization and radiationhydrodynamic modelling. We discuss the results in Sect. 4, and summarize our conclusions in Sect. 5 .

\section{HST/WFPC2 data}

The HST archive images are available from the HST archival data centre. The [O III] 4363 and 5007 emissionline images can be used to find an [O III] electron temperature map. We searched the archive for WFPC2 images of $[\mathrm{O} I I I]$ and $\mathrm{H} \alpha / \mathrm{H} \beta$. As will be explained below, we need an $\mathrm{H} \alpha$ or $\mathrm{H} \beta$ image to eliminate the continuum contribution from the [O III] images. The $H S T$ archive contains 11 images of NGC 6543 in these filters. After careful examination, we selected the three best ones: U27Q0103T ([O III] 4363); U27Q010FT (H $\alpha$ ); and U27Q010AT ([O III] 5007). The central part of the [O III] 4363 image is saturated by the central star. See Table 1 for the details.

\subsection{Reduction of the WFPC2 images}

The archived WFPC2 images have already been calibrated via the pipeline procedure, i.e. the analog-to-digital correction, bias level removal, bias image subtraction, dark image subtraction, flat-field multiplication, and shutter shading correction. Using standard IRAF routines, we removed the cosmic rays. The retrieved images were converted into flux units (or magnitudes) using the header information.
Table 2. WFPC2 narrow band filter set.

\begin{tabular}{llll}
\hline \hline Name $($ Ion $)$ & Width $(\AA)$ & Peak $(\lambda)^{1}$ & $Q(20 \AA)^{2}$ \\
\hline F437N $([\mathrm{O}$ III $])$ & 25.2 & $49.6(4368)$ & $86.4 \%$ \\
F502N $([\mathrm{O}$ III $])$ & 26.9 & $57.9(5009)$ & $105.0 \%$ \\
F656N $(\mathrm{H} \alpha)$ & 21.9 & $86.2(6561)$ & $112.3 \%$ \\
\hline
\end{tabular}

${ }^{1}$ Peak transmission efficiency in $\%$ (wavelength $\lambda$ at peak). ${ }^{2}$ The transmitted light relative to an ideal $20 \AA$ bandwidth filter, assuming this ideal filter has $100 \%$ transmission over a with $20 \AA$ bandwidth.

\subsubsection{Filter bandwidth correction}

The width and transmission efficiency of the three filters differ (see Table 2). To compare the images, one must correct for these differences. We use a normalizing function to obtain the observed photon flux within a bandwidth of $20 \AA$ :

$F_{\text {norm }}=F_{\mathrm{HST}} / Q(20 \AA)$,

where $F_{\mathrm{HST}}$ is the calibrated final flux; $Q(20 \AA)$ (given in the last column of Table 2) is the transmission efficiency for an ideal 20 Å bandwidth filter with $100 \%$ transmission. Once $F_{\text {norm }}$ has been obtained for each image, we may compare them with each other.

\subsubsection{Interstellar extinction}

The interstellar extinction is corrected using the relation

$F_{\mathrm{e}-\mathrm{c}}=F_{\text {norm }} \times 10^{C \cdot k_{\lambda}}$,

where $C$ is the logarithmic extinction coefficient at $\mathrm{H} \beta$, and $k_{\lambda}$ the extinction parameter from Seaton (1979). $C(\mathrm{H} \beta)$ values of $0.18,0.20$ and 0.30 have been presented by Kaler (1976), Middlemass et al. (1989), and Hyung et al. (2000), respectively. In what follows we will adopt $C(\mathrm{H} \beta)=0.20$. The uncertainty in the adopted $C$ value does not affect the results derived in this paper.

\subsubsection{Continuum correction}

The filter widths are $\sim 20 \AA$, while the full width at half maximum $(F W H M)$ of the emission lines is only $\sim 1 \AA$. Thus, the images can contain a significant contribution from continuum photons. Since no offset nebular filter images are available in the $H S T$ archive, and the continuum is mostly produced by hydrogen quanta (see below), we used the $\mathrm{H} \alpha$ WFPC2 image to correct for the continuum contribution in the [O III] filter images.

First, we estimated the contribution to the continuum of $\mathrm{H}$ recombination plus bremsstrahlung, assuming an electron temperature of $10000 \mathrm{~K}$ and $\mathrm{He} / \mathrm{H}$ abundance ratios of $N\left(\mathrm{He}^{+}\right) / N\left(\mathrm{H}^{+}\right)=0.1$ and $N\left(\mathrm{He}^{++}\right) / N\left(\mathrm{H}^{+}\right)=$ 0.01 . We find this contribution to be $92.7 \%$ near [O III] 4363 emission line, and 91.2\% near [O III] 5007. The theoretical continuum-emission coefficients have been 

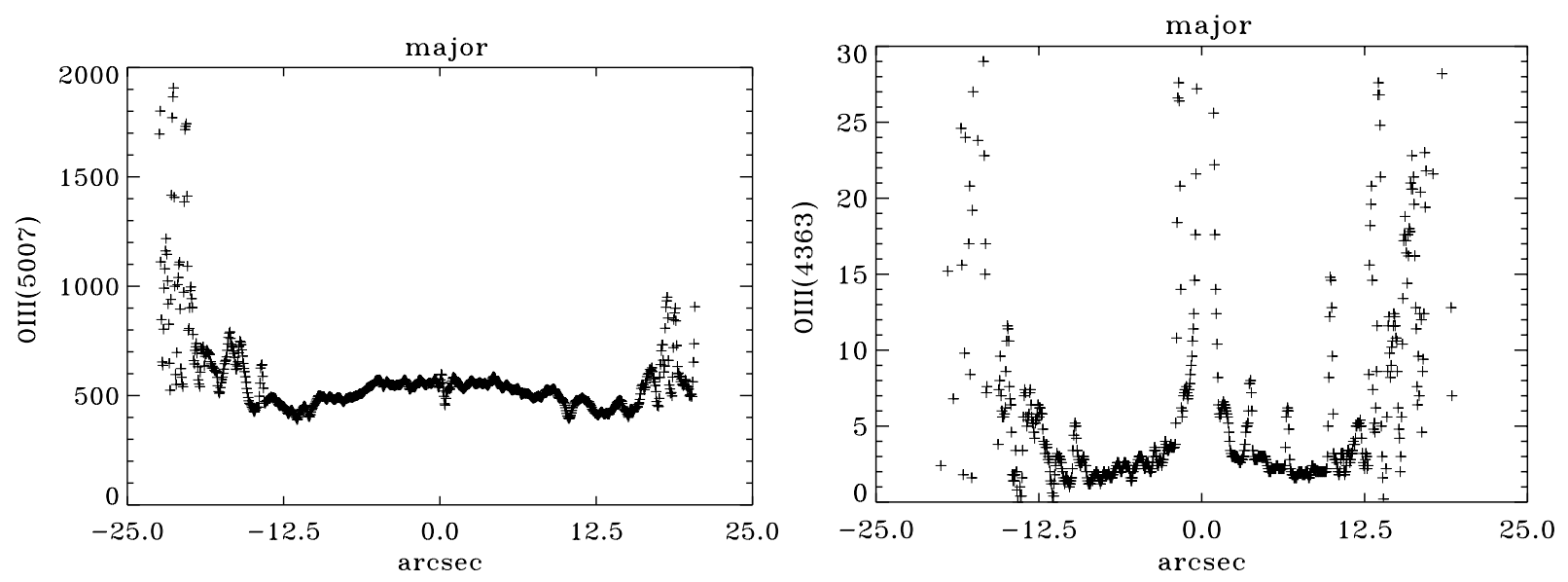

Fig. 1. [O III] intensities along the major axis [scaled to $I(\mathrm{H} \beta)=100]$. On the left $I([\mathrm{O} I I I] 5007)$, on the right $I([\mathrm{O}$ III $] 4363)$.

adopted from Brown \& Mathews (1970); see their Tables $1-5$. This simple calculation shows that hydrogen emission dominates the continuum at the wavelengths of interest.

The continuum-corrected [O III] images can then be obtained from the following relations,

$F_{\mathrm{f}}(5007)=F_{\mathrm{e}-\mathrm{c}}(5007)-F_{\mathrm{e}-\mathrm{c}}(\mathrm{H} \alpha) \times A(5007)$,
$F_{\mathrm{f}}(4363)=F_{\mathrm{e}-\mathrm{c}}(4363)-F_{\mathrm{e}-\mathrm{c}}(\mathrm{H} \alpha) \times B(4363)$,

where $A(5007)$ and $B(4363)$ are the extinction-corrected continuum fluxes near $5007 \AA$ and $4363 \AA$, respectively, divided by the extinction-corrected continuum flux near the $\mathrm{H} \alpha$ line + the $\mathrm{H} \alpha$ emission flux itself. Values for $A$ and $B$ were derived from our ground-based Hamilton Echelle Spectrograph (HES) data (Hyung et al. 2000): $A(5007) \simeq 1.21 \times 10^{-3}$ and $B(4363) \simeq 5.28 \times 10^{-3}$. Aller (1984) lists the theoretical values as $A(5007)=$ $3.1 \sim 4.4 \times 10^{-3}$ and $B(4363)=3.5 \sim 6.2 \times 10^{-3}$, and also shows that $B(4363) / A(5007) \sim 1.2$. Our $B$ value agrees with this within $20 \%$, but we find a 3 to 4 times smaller value for $A(5007)$. This deviation is probably caused by an overcorrection for scattered light in analysis of the HES data. As a test we also derived the values for the bright core nebula, and there our procedure does reproduce the expected theoretical values. However, the strength of the [O III] 5007 is such that an error of a factor of four in $A(5007)$, only affects the derived intensity to less than $0.5 \%$. An error of about $20 \%$ in $B(4363)$ also does not influence our results.

Although the ratio of continuum to line emission may have positional variations, we assume that $A$ and $B$ remain constant throughout the nebula and halo. This assumption is unavoidable, due to the unavailability of offset continuum filter images. It may be not a bad assumption, since the stratification effect of the $\mathrm{H} \alpha$ (or $\mathrm{H} \beta$ ) recombination image is generally not conspicuous, compared with that of the line images, e.g. [O III] 5007 or [N II] 6548. The F437N image may suffer from some light contamination by $\mathrm{H} \gamma$ at $4341 \AA$.
Table 3. [O III] line intensities.

\begin{tabular}{cccc}
\hline \hline Name (Ions) & Core (North) & Core (East) & Halo $^{2}$ \\
\hline [O III] 4363 & 2.05 & 1.58 & 28 \\
[O III 5007 & 704 & 518 & 1528 \\
\hline
\end{tabular}

${ }^{1}$ Hyung et al. (2000).

${ }^{2}$ MCW89.

Note: Extinction corrected intensities are given based on $I(\mathrm{H} \beta 4861)=100(C=0.2,0.3$ for $H S T$ and HES, respectively). See the main text.

In order to reduce the scatter, we rebinned the image from $0^{\prime \prime} 1 \times 00^{\prime \prime} 1$ to $0.3 \times 00^{\prime \prime} .3$ pixels. Figure 1 displays the [O III] 5007 and 4363 line intensities on a flux scale where $I(\mathrm{H} \beta)=100$. Note how the [O III] 4363 intensities within $\sim 2^{\prime \prime}$ of the centre are strongly affected by the saturation caused by the central star.

Table 3 lists the spectroscopic line intensities of the core H II region (Hyung et al. 2000) and the outer halo (MCW89). A comparison with Fig. 1 shows that, within the uncertainties, the correspondence between the spectroscopic data and the images is reasonable.

\subsection{4. [O III] temperature map}

Figure 2 shows the temperature maps for three different electron temperatures ranges: $T_{\mathrm{e}}=8000-9000 \mathrm{~K}$; $11000-12000 \mathrm{~K}$; and $13000-14000 \mathrm{~K}$, respectively. As was found in previous ground-based studies, the electron temperature of the core nebula is $8000-9000 \mathrm{~K}$. The $11000-12000 \mathrm{~K}$ image clearly displays an ellipsoidal envelope surrounding the core nebula. Figure $2 \mathrm{c}$ shows that the inner halo region contains hotter material of $T_{\mathrm{e}} \sim 14000 \mathrm{~K}$. The temperature in the very central region also appears to be high. However, those values are suspect, due to the saturation by the central star in the [O III] 4363 image.

In Fig. 3, we plot the electron temperature distribution along the major and minor axes, and at an intermediate 
angle. The position angles are $-32^{\circ},+58^{\circ}$, and $+13^{\circ}$, respectively.

We estimated the errors in Figs. 2 and 3 using a qualitative analysis. We found them to be less than $\triangle T_{\mathrm{e}} \sim$ $500 \mathrm{~K}$ in the core nebula, and at least three times larger in the inner halo, $\simeq 1500 \mathrm{~K}$. This latter region could be partly filled with relatively low temperature gas, like that seen in Fig. 2a. In this case, the scatter in Fig. 3 may reflect real temperature fluctuations involving geometrical irregularities. Otherwise, it would be due to errors of around $3000 \mathrm{~K}$ in the halo.

Figure 3 shows a number of local peaks with FWHM $\sim 0^{\prime \prime} 3-0^{\prime \prime} .4$, where the electron temperature increases sharply. These could be due to blobs or filaments. We compared temperature profiles, e.g. 0". 8 apart, parallel to the major and minor axes. No structure survived in the next adjacent scan; apparently, blobs substantially larger than 0. . 4 do not exist. This leads to the conclusion that any existing substructures must be about 0 .'4 (0.002 pc for an assumed distance of $1 \mathrm{kpc}$ ), or less.

Figure 3 also shows third order Legendre polynomial curves fitted to the radial electron temperature distribution (excluding the central region). These fits suggest that the maximum electron temperature in the halo occurs at a radial distance of $r \sim 0.09 \mathrm{pc}\left(18^{\prime \prime}, 17^{\prime \prime}\right.$, and $14^{\prime \prime}$, respectively), beyond which it falls off again. The radial distribution of the HST electron temperature suggested by the polynomial fitting indicates a high electron temperature location, which may roughly form a complete circle of $r \sim$ $0.09 \mathrm{pc}$. The halo temperature fitting found from the $H S T$ images may be the projection of a relatively large shell, of $r \sim 0.09-0.1 \mathrm{pc}$.

\section{Excitation by hardened UV or shock?}

The thermal properties of the gas in the halo are set by photo-ionization heating, and also possibly by shock heating. We will now investigate whether these two processes can explain the observed electron temperatures.

\subsection{Hardening of the UV radiation field}

Long ago, Aller et al. (1939) suggested that a hardening of the UV radiation field from the central star might be responsible for a rise of $T_{\mathrm{e}}$ in the outer part of photoionized PNe. Photoionization modeling by Hyung et al. (2000) suggested that the electron temperature near the outer boundary (at $0.056 \mathrm{pc}$ ) of the core H II region of NGC 6543 is higher than that of the inner region (at 0.05 pc) by $600 \mathrm{~K}$, due to a hardening of far UV stellar radiation. Can this hardened stellar UV radiation also raise the electron temperature of the halo gas to the value suggested by the HST [O III] images? MCW89 showed that the emission in the outer halo could not be produced by photo-electric heating: they were only able to reproduce a halo temperature some $2000 \mathrm{~K}$ above the core region temperature.
We extend the photoionization model by Hyung et al. (2000) to see whether the hardened UV radiation from the WR-type central star produces the high electron temperatures in the inner halo, or gives the observed [O III] 5007/4363 line ratios. Note that the photoionization model by Hyung et al. (2000) indicates that NGC 6543's bright core is density bounded. Thus, there are still plenty of hard, far-UV photons available to ionize the gas in the halo region. Since the relatively low density $\left(35 \mathrm{~cm}^{-3}\right)$ outer halo case was already tested by MCW89, we explore higher densities for the inner halo gas. The description of the photoionization model, the improved Sobolev approximation (ISA) model atmosphere for the hydrogen deficient Wolf Rayet type central star of the planetary nebula (CSPN), and references to selected atomic parameters may be found in Hyung et al. (2000). We adopted the abundances from the core nebula, as described by Hyung et al. (2000). The results show that a higher electron temperature can only be achieved for very high densities. For, example, the model with $N_{\mathrm{H}}=10^{5} \mathrm{~cm}^{-3}$ and a small filling factor, $f=0.0001$, gives $I(4959+5007) / I(4363)=$ 105.8 (corresponding to $\left.T_{\mathrm{e}} \sim 12000 \mathrm{~K}\right)$. Since the spectroscopic observations indicate that the electron density in the halo is low, this high density model is not an appropriate solution to the halo emission. Nonetheless, there may be some implications for the physical conditions of the filaments observed in the central or halo region of some $\mathrm{PNe}$ - the filaments are density enhanced structures, in any case.

\subsection{Shock heating}

An alternative way to heat gas is through shocks. Shocks are caused either by pressure or velocity differences. In order to produce a noticeable temperature increase of the order of $15000 \mathrm{~K}$, the shock speed needs to be at least $20 \mathrm{~km} \mathrm{~s}^{-1}$. One origin for such shocks would be a "leaky" stellar wind bubble. If the material which the fast wind is running into is quite clumpy, a mass-loaded flow would proceed beyond the core nebula into the halo region, where it would interact with halo material to produce the necessary shocks. This is the model favoured by Meaburn et al. (1991) to explain the measured electron temperatures in the outer halo. Whereas the structures in the outer halo also morphologically suggest such an interaction, the inner halo looks much smoother, and shows no signs of interacting with a mass-loaded flow from the core. In fact, with the parameters given in Meaburn et al. (1991), the density in the inner halo would be dominated by the massloaded flow. In support of the mass-loaded flow model, we should mention that the images presented in BWH01 do reveal radial structures in the inner halo, which mostly (but not uniquely) appear to originate from the FLIER regions in the core nebula. If these are extensions of a faster outflow in the FLIERs, and not illumination effects, they could certainly produce (local) shocks in the inner halo. 

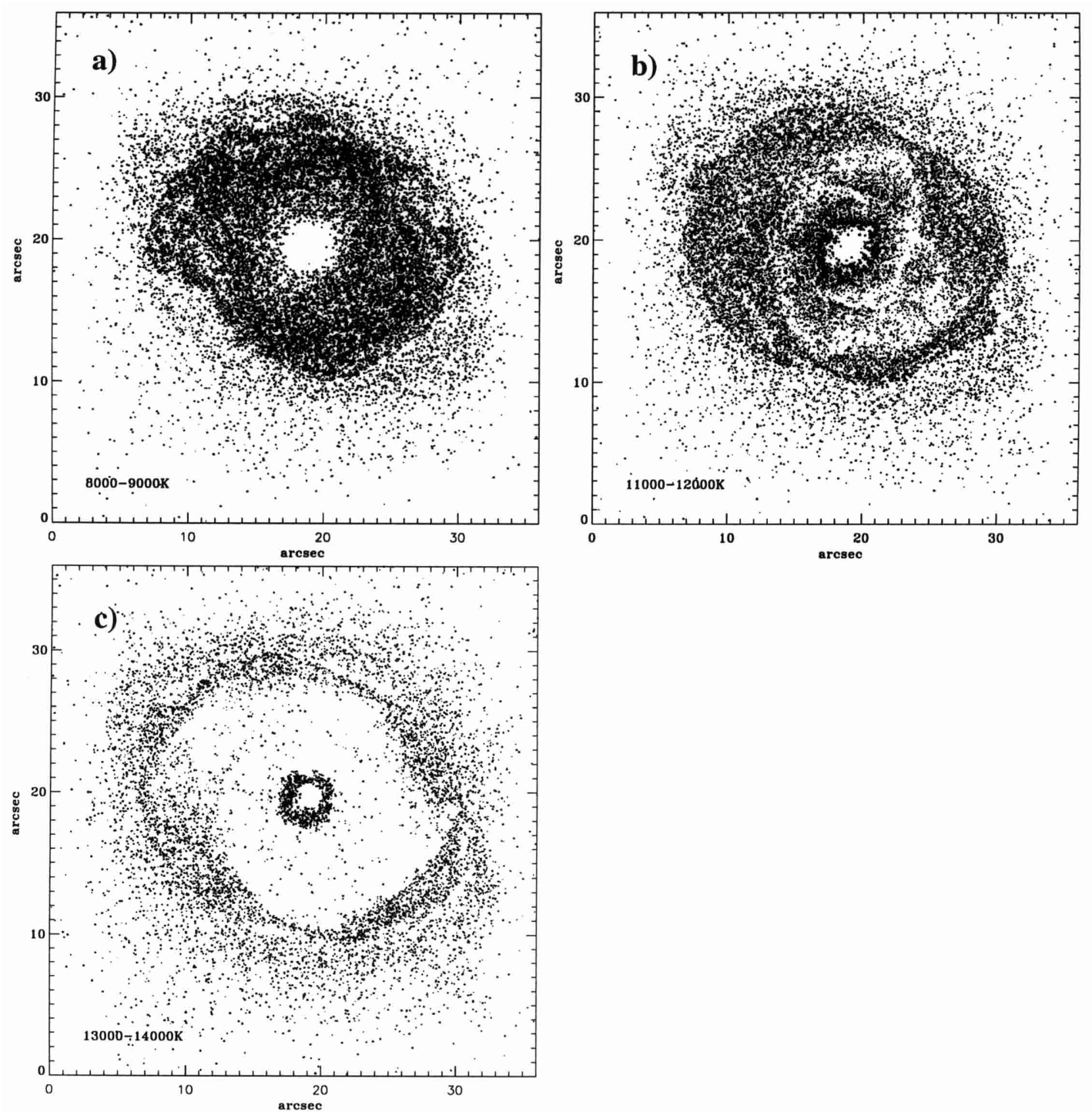

Fig. 2. [O III] temperature maps for the ranges a) $\left.\left.T_{\mathrm{e}}([\mathrm{O} \mathrm{III}])=8000 \sim 9000 \mathrm{~K}, \mathbf{b}\right) T_{\mathrm{e}}([\mathrm{O} \mathrm{III}]) 11000 \sim 12000 \mathrm{~K}, \mathbf{c}\right) T_{\mathrm{e}}([\mathrm{O} \mathrm{III}])$ $13000 \sim 14000 \mathrm{~K}$. An upper limit of $20000 \mathrm{~K}$ has been set for the area around the CSPN.

On the other hand, the presence of the rings in the inner halo region already indicates that there are density and hence pressure differences there. The idea that the inner halo is dynamic is strengthened by the increase in the line widths in the inner halo region to about $30 \mathrm{~km} \mathrm{~s}^{-1}$ (Bryce et al. 1992; BWH01), which could be explained by a superposition of faster moving shells. In this case the shells would have velocities well in excess of the $5 \mathrm{~km} \mathrm{~s}^{-1}$ derived for the outer halo. If the shells were created while the CSPN was on the AGB, velocity differences would also have helped in their survival up to the current era. Density variations without velocity variations tend to be smoothed out. If the shells are produced by the mechanism explained in Simis et al. (2001), velocity differences of $50 \mathrm{~km} \mathrm{~s}^{-1}$ or higher can be reached (Simis, private communication).

It will be difficult to measure the true expansion velocity of the shells, since the slit position which would capture it the best, would also contain the bright and kinematically complicated core nebula (see Miranda \& Solf 1992).

\subsubsection{Numerical model}

In order to show the feasibility of shock heating by the shells, we set up a simple numerical experiment. For this we use a hydrodynamic code coupled to a photo-ionization calculation. The hydrodynamic part of the code is based on Roe's approximate Riemann solver (Mellema 1993; Eulderink \& Mellema 1995). In each numerical time step, the heating and cooling rates are calculated using the DORIC routines (Raga et al. 1997; Mellema et al. 1998), which calculate the non-equilibrium ionization, heating and cooling of the gas. Since we are not concerned with the shaping of the entire nebula, and want to resolve the postshock cooling region, we consider only the one-dimensional (spherical) case. For this we used 12000 computational 

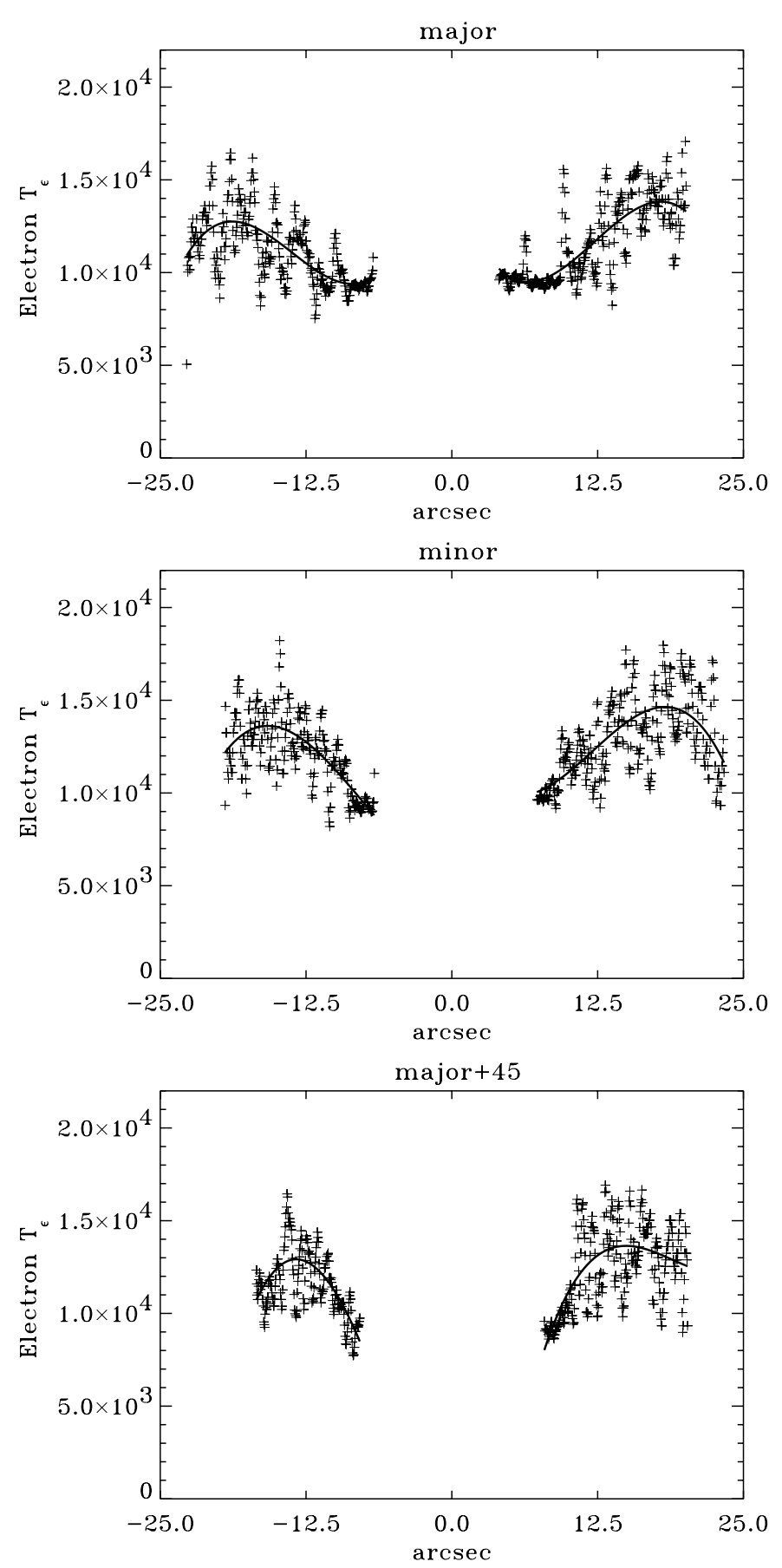

Fig. 3. [O III] electron temperatures in K: from top to bottom: along the major axis, along the minor axis, and in between the major and minor axes (at major $+45^{\circ}$ ). Since binning of $3 \times 3$ pixels was applied, the spatial resolution is $0^{\prime \prime} 3$. We exclude the region near the CSPN.

cells of size $2 \times 10^{11} \mathrm{~cm}$. At this cell size, the CourantFriedrichs-Lewis (CFL) time step imposed by the hydrodynamics is much smaller than the cooling time, and all cooling regions are well resolved.

The optical radius of the main core is about $10^{\prime \prime}$, while the halo region shown in the $H S T$ images corresponds to an area with a radius of about $30^{\prime \prime}$, which is $0.15 \mathrm{pc}$.
Table 4. Input parameters for the simulation.

\begin{tabular}{ll}
\hline \hline$\dot{M}_{\text {low }}$ & $4 \times 10^{-6} M_{\odot} \mathrm{yr}^{-1}$ \\
$v_{\text {low }}$ & $40 \mathrm{~km} \mathrm{~s}^{-1}$ \\
$\dot{M}_{\text {high }}$ & $2 \times 10^{-5} M_{\odot} \mathrm{yr}^{-1}$ \\
$v_{\text {high }}$ & $80 \mathrm{~km} \mathrm{~s}^{-1}$ \\
$\tau_{\text {variation }}$ & $200 \mathrm{years}^{\prime \prime}$ \\
$r_{\text {in }}$ (boundary) & $0.05 \mathrm{pc}\left(10^{\prime \prime}\right)$ \\
$r_{\text {out }}$ (boundary) & $0.15 \mathrm{pc}\left(30^{\prime \prime}\right)$ \\
$\Delta r$ & $6.48 \mu \mathrm{pc}$ \\
Central Star $T_{\text {eff }}$ & $48000 \mathrm{~K}$ \\
Central Star $L$ & $3510 L \odot$ \\
\hline
\end{tabular}

Thus, we set the inner radius of the computational grid to be $0.05 \mathrm{pc}$, and let the grid run out to $0.15 \mathrm{pc}$.

At the start of a simulation, the value of the electron temperature is given by the equilibrium temperature provided by the photo-ionization model.

For the abundances, we used the values derived by Hyung et al. (2000) for the core nebula: $\mathrm{He} / \mathrm{H}=0.13$, $\mathrm{C} / \mathrm{H}=5.0(-5), \mathrm{N} / \mathrm{H}=1.2(-4), \mathrm{O} / \mathrm{H}=3.0(-4)$, $\mathrm{Ne} / \mathrm{H}=5.0(-5), \mathrm{S} / \mathrm{H}=7.0(-6)$.

Bryce et al. (1992) found the outer halo to be kinematically inert, with an upper limit to the expansion velocity of $4.6 \mathrm{~km} \mathrm{~s}^{-1}$. The observed electron density of the halo region obtained from the [O II] diagnostics can set a constraint for the mass loss rate of the slow wind. Middlemass found $35_{-35}^{100} \mathrm{~cm}^{-3}$ (in the outer halo, outside the HST image). Taking a (pre-shock) number density of $n_{\mathrm{H}} \sim 20 \mathrm{~cm}^{-3}$ at $0.15 \mathrm{pc}$, we arrive at a mass loss rate for the slow wind of $\dot{M}_{\text {slow }}=10^{-6} M_{\odot} \mathrm{yr}^{-1}$. Alternatively, BWH01 find a total mass of $\sim 0.1 M_{\odot}$ ejected between 17500 and 6500 years ago (assuming a slow wind velocity of $10 \mathrm{~km} \mathrm{~s}^{-1}$ ), which gives a mass loss rate of $\sim 10^{-5} M_{\odot} \mathrm{yr}^{-1}$. For a slower wind velocity of $5 \mathrm{~km} \mathrm{~s}^{-1}$, one obtains half this value. We take a base slow wind mass loss rate of $10^{-6} M_{\odot} \mathrm{yr}^{-1}$.

At the inner edge of the grid, we then vary the mass loss rate between this base value and $10^{-5} M_{\odot} \mathrm{yr}^{-1}$, and with a velocity difference of $40 \mathrm{~km} \mathrm{~s}^{-1}$. The variation is sinusoidal with a period of 200 years.

The typical sound speed in an ionized gas is around $10 \mathrm{~km} \mathrm{~s}^{-1}$. This means that if we choose a base inflow velocity of 5 or $10 \mathrm{~km} \mathrm{~s}^{-1}$, the inflow will be close to sonic. In this case a steady wind will quickly accelerate (similar to a Parker wind solution) and achieve supersonic velocities. In practice this means that a fixed inner boundary inflow velocity of around $10 \mathrm{~km} \mathrm{~s}^{-1}$ cannot be used. In previous simulations (see e.g. Mellema 1994), this was never a problem, since the inflow velocity was the highly supersonic fast wind. Here, where we want to concentrate on the halo, this physical behaviour poses a problem. To get around this, we decided to use inflow velocities which are well over sonic. In practice this means a base inflow velocity of $40 \mathrm{~km} \mathrm{~s}^{-1}$, and consequently a peak inflow velocity of $80 \mathrm{~km} \mathrm{~s}^{-1}$. We scaled the mass loss rates up by a factor of 4 and 2, to achieve roughly the same densities as before. 

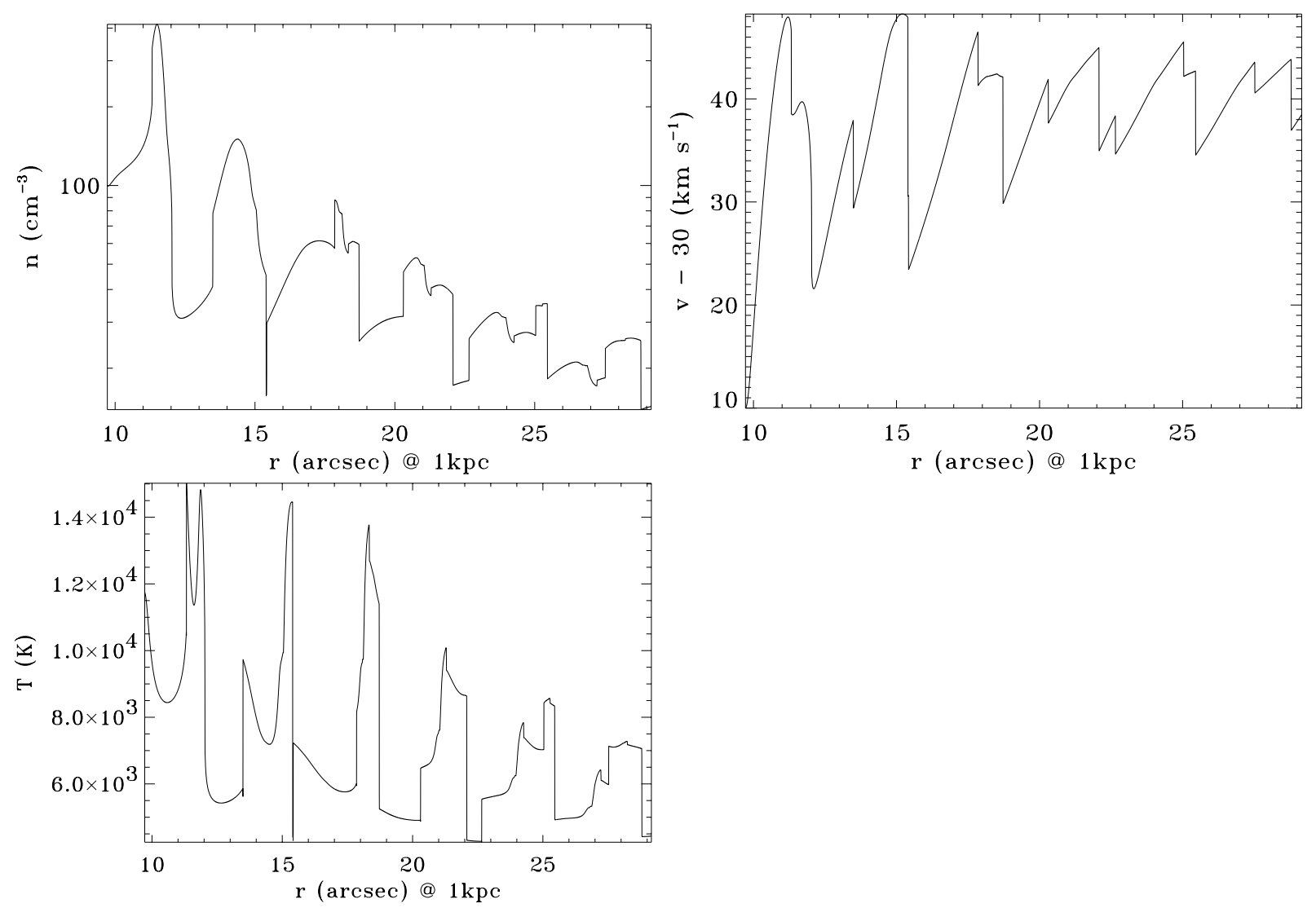

Fig. 4. Hydrodynamics simulation. Graphs showing the number density $(n)$, velocity $(v)$, and temperature $(T)$. The time is 2550 years.

For the photo-ionization calculation we assumed the column densities at the inner grid edge to be $10^{12} \mathrm{~cm}^{-2}$ for $\mathrm{HI}$ and HeI, and $10^{15} \mathrm{~cm}^{-2}$ for He II. The parameters adopted in the simulation are listed in Table 4.

\subsubsection{Results of the simulation}

Figure 4 shows plots of the density, velocity and temperature at 2550 years after the start of the simulation. The situation shown is the periodic solution which develops after roughly 1200 years. One sees the result of a series of pulses, which are interacting and moving out. One should realise that the faster parts of a pulse will run ahead of the slower ones, and may be overtaken by the next pulse, leading to complicated structures as a function of distance. The velocity shows a "saw-tooth" profile, which is still slightly accelerating outward.

Since our hydrodynamic model includes a photoionization model, we are able to construct the $I(5007) / I(4363)$ ratio from the local electron density, temperature, and $\mathrm{O}^{2+}$ density. We calculated the emission, turned it into a three-dimensional distribution and projected this on the sky. In Fig. 5 we show the [O III] 5007+4959 intensity, both as function of radius, and along a radial cut through the image. One sees that the image shows the typical emission characteristics of projected shells, just as seen in the observations, cf. BWH01.

Figure 5 also shows the electron temperature derived from the $I(5007+4959) / I(4363)$ ratio in the projected images, as well as on a cut through a projected temperature map weighted with the $\mathrm{H}$ recombination emission. All image data were smoothed with a Gaussian of $F W H M$ of 0 '” 3 . The synthesized data can reproduce the electron temperatures found in the HST results, including the drop in temperature as function of radius. The projection actually tends to hide the biggest variations in the line ratio, which is due to the small filling factor of the areas of the highest temperature. Comparing the cuts through the two temperature maps, one can see how the [O III] ratio does give higher temperatures, as seen in the case of the so-called "temperature fluctuations". Note also how there actually is a slight displacement between the high temperature peaks in both images. This is because the $\mathrm{H}$ recombination-weighted image has a much stronger bias for areas of higher density.

In Fig. 6, we illustrate the effect of the velocity variations by showing synthesized line shapes at four different positions. The velocities from the simulation were decreased by $30 \mathrm{~km} \mathrm{~s}^{-1}$, and then smoothed both along the velocity $\left(F W H M 10 \mathrm{~km} \mathrm{~s}^{-1}\right)$ and positional $\left(2^{\prime \prime}\right)$ directions in order to simulate the groundbased observations. 

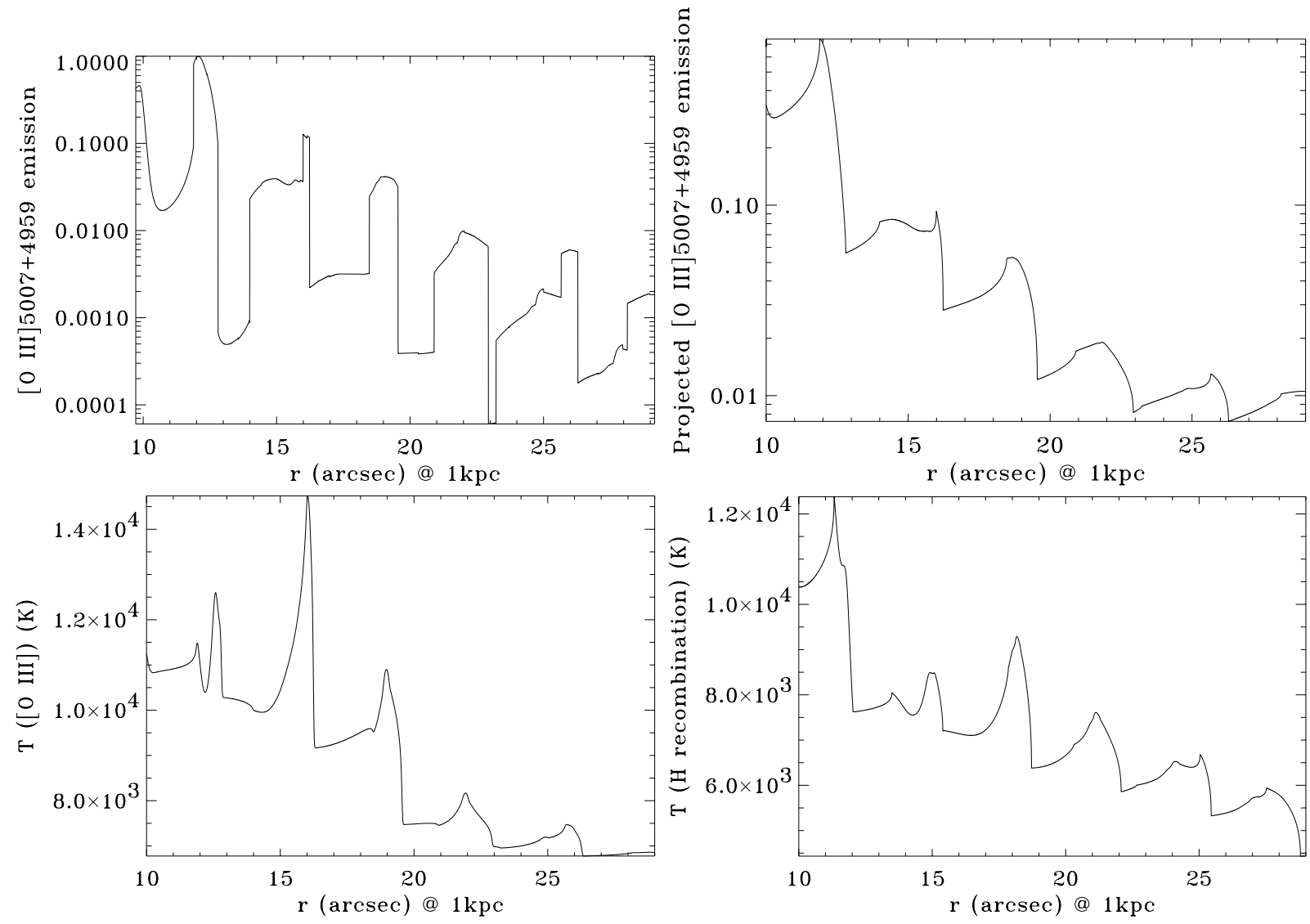

Fig. 5. Top left: [O III] $(5007+4959)$ intensity as a function of radius, in arbitrary units. Top right: the same but projected on the sky. Bottom left: the electron temperature derived from the [O III] $(5007+4959) / 4363$. Bottom right: the electron temperature derived from $\mathrm{H}$ recombination; also compare these temperatures to those in Fig. 4.

One sees how, at all four positions, the profiles are quite wide, consistent with the observations. However, they are not Gaussian; the fact that they orginate from different shells is still visible. Since no line shapes have been published, it is hard to say how far our calculations deviate from the observations.

\section{Discussion}

The results of the simulation show that the interaction between mass loss variations can reproduce shells which match the observed ones morphologically, and to a large extent also kinematically and thermally. This is the first attempt at modelling properties of the shells, other than their appearance. However, both the observational data and the modelling need improvement, and the results presented here should be viewed as a simple, first attempt model.

To start with the observations, narrowband images are not the best method for measuring line ratios accurately, and a closer look at the kinematic data would not hurt either. It would also be very interesting to see if similar effects are found in other $\mathrm{PNe}$ with surrounding rings. Unfortunately, the ideal observations would be difficult to carry out; to resolve the individual shells, one needs the $H S T$, but in order to do the spectroscopy on these faint shells one needs a large aperture. Also, the velocity resolution of the STIS instrument on board HST is insufficient for a proper analysis of the line shapes.

The shortcomings of the numerical model are obvious. For one, we were forced to introduce an unrealistically high inflow velocity, and we set the variations to be sinusoidal, which is hardly what one would expect. Ideally one would like to follow the mass loss variations while the star is still on the AGB, and let the different phases interact among themselves, and then study the effect of photo-ionization when the central star evolves into a post-AGB star. One would also like the mass loss from the star to vary with its properties, so that a faster post-AGB wind starts to interact with the inner parts of the AGB wind. This would solve the problem of the subsonic inflow condition, as well as the question of the column density of the core nebula. In short one would model the evolution of the circumstellar material from the (late) TP-AGB until the $\mathrm{PN}$ phase. This has, for example, been done by Steffen \& Schönberner (2000), but their results do not address the points raised in this paper. We plan to modify our simulations in order to model mass loss variations during the AGB phase, and also their fate after the star evolves off the AGB.

A further complication is the relation between the inner halo/ring area and both the core nebula and outer halo. Compared to these two structures, the inner halo 

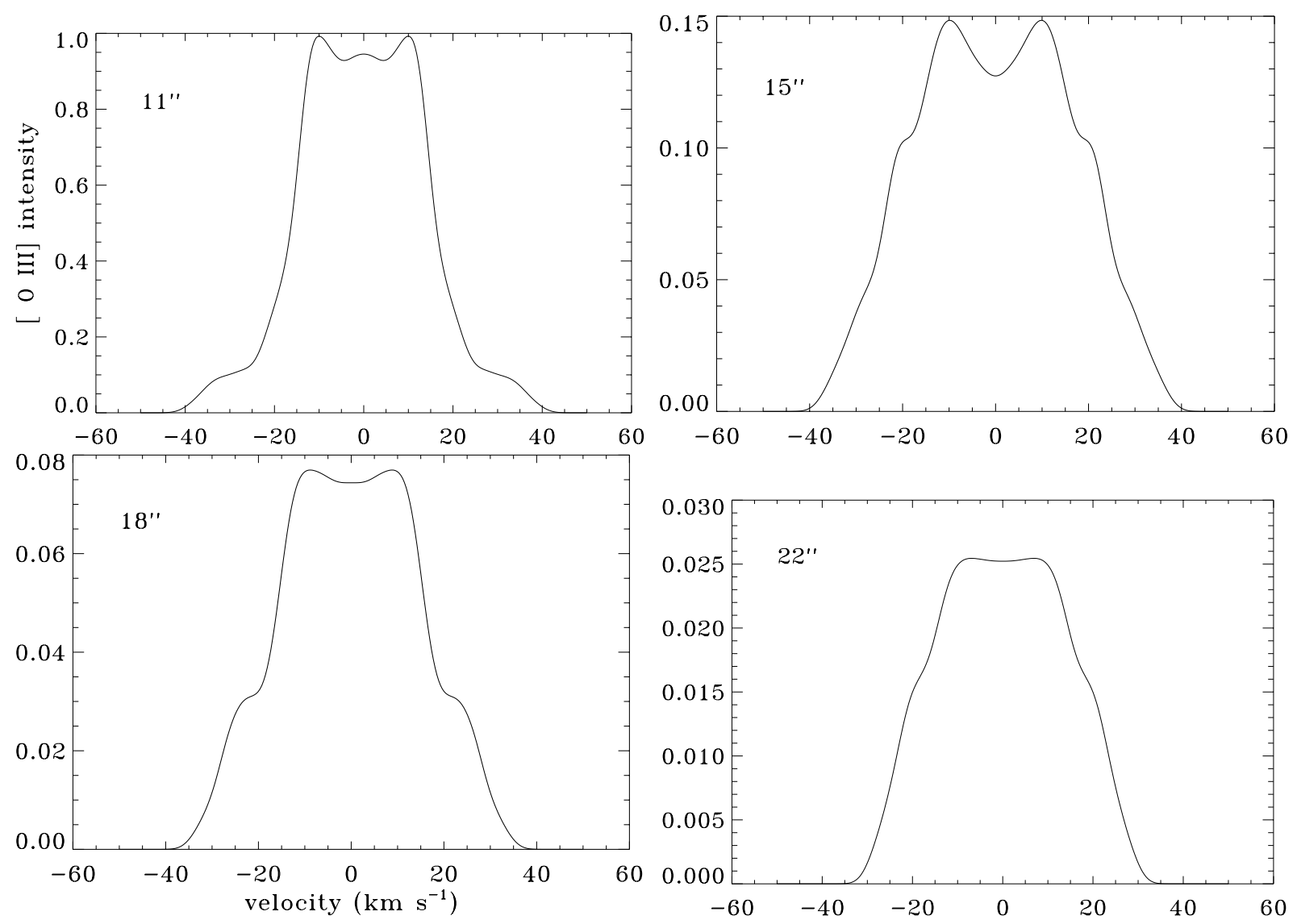

Fig. 6. Line shapes for [O III] 5007 at $11^{\prime \prime}, 15^{\prime \prime}, 18^{\prime \prime}$, and $22^{\prime \prime}$ from the central star (for a distance of $1 \mathrm{kpc}$ ). The data has been convolved with a Gaussian to achieve $10 \mathrm{~km} \mathrm{~s}^{-1} F W H M$ velocity resolution, and also $2^{\prime \prime}$ spatial resolution.

seems an oasis of simplicity. The Chandra data have shown that the core nebula consists of an inner wind-swept elliptical nebula, surrounded by a (bipolar?) structure of lower ionization, pierced by FLIERs or jets along the major axis. The chaotic structure of the outer halo also points to some dynamic interaction, altough this material was presumably lost long before the inner halo and core nebula. The complete story of NGC 6543 has not yet been finalized.

Still, it may be that the inner halo is indeed a relatively quiet area of material lost just before the star turned off the $\mathrm{AGB}$, in a period in which the mass loss was variable in density and velocity, something which according to some mass loss models is more likely to happen at the higher effective temperatures and luminosities towards the end of the AGB (Simis, private communication). The numerical study presented above shows that such a model can explain the properties of the "rings around the Cat's Eye".

\section{Conclusions}

We analyze archival HST [O III] 4363 and $5007 \AA$ narrowband images and find an increased inner halo electron temperature of $\sim 15000 \mathrm{~K}$ for NGC 6543 . This higher temperature is found in the same region as the "rings around the Cat's Eye" reported by BWH01. This higher electron temperature cannot be explained by photo-ionization models, and we conclude that they must be produced by shocks. We show that, in principle, velocity and mass loss variations in the slow AGB wind, required for the production and survival of the rings, would also be able to explain the higher electron temperature in the inner halo, as well as the observed higher velocity dispersion in that region.

Acknowledgements. Some of the data presented in this paper was obtained from the Multimission Archive at the Space Telescope Science Institute (MAST). STScI is operated by the Association of Universities for Research in Astronomy, Inc., under NASA contract NAS5-26555.

This research was supported in part by the KRF No. 2000015-DP0445, by the Korea MOST Grant Star No. Star 002-500-00, and by the KOSEF Grant No. 2000-1-113-001-5 to KAO. SH is thankful to staff of the KISTI for permission to use their Compaq GS320 supercomputer. We also thank the referee Dr. M. Peimbert, for a careful review and valuable suggestions and Dr. A. Fletcher (KAO) for help with the preparation of this paper.

The research of GM has been made possible by a fellowship of the Royal Netherlands Academy of Arts and Sciences.

\section{References}

Aller, L. H. 1984, Physics of Thermal Gaseous Nebulae (Dordrecht: Reidel Publisher), 105

Aller, L. H., Baker, J. G., \& Menzel, D. H. 1939, ApJ, 90, 601 
Balick, B., Wilson, J., \& Hajian, A. R. 2001, AJ, 121, 354 (BWH01)

Brown, R. L., \& Mathews, W. G. 1970, ApJ, 160, 939

Bryce, M., Meaburn, J., Walsh, J. R., \& Clegg, R. E. S. 1992, MNRAS, 254, 477

Castor, J. I., Lutz, J. H., \& Seaton, M. J. 1981, MNRAS, 194, 547

Chu, Y.-H., Guerrero, M. A., Gruendl, R. A., Williams, R. M., \& Kaler, J. B. 2001, ApJ, 553, L67

Eulderink, F., \& Mellema, G. 1995, A\&AS, 110, 587

García-Segura, G., López, J. A., \& Franco, J. 2001, preprint [astro-ph/0104154]

Harpaz, A., Rappaport, S., \& Soker, N. 1997, ApJ, 487, 809

Hyung, S., Aller, L. H., Feibelman, W. A., Lee, W.-B., \& de Koter, A. 2000, MNRAS, 318, 77

Kaler, J. B. 1976, ApJS, 31, 517

Mastrodemos, N., \& Morris, M. 1999, ApJ, 523, 357

Mauron, N., \& Huggins, P. J. 2000, A\&A, 359, 707
Meaburn, J., Nicholson, R., Bryce, M., Dyson, J. E., \& Walsh, J. R. 1991, MNRAS, 252, 535

Mellema, G. 1993, Ph.D. Thesis: Numerical models for the formation of aspherical planetary nebulae (Leiden University)

Mellema, G. 1994, A\&A, 290, 915

Mellema, G., Raga, A. C., Canto, J., et al. 1998, A\&A, 331, 335

Middlemass, D., Clegg, R. E. S., \& Walsh, J. R. 1989, MNRAS, 239, 1 (MCW89)

Miranda, L. F., \& Solf, J. 1992, A\&A, 260, 397

Raga, A. C., Mellema, G., \& Lundqvist, P. 1997, ApJS, 109, 517

Sahai, R., Trauger, J. T., Watson, A. M., et al. 1998, ApJ, 493, 301

Seaton, M. J. 1979, MNRAS, 187, 73

Simis, Y. J. W., Icke, V., \& Dominik, C. 2001, A\&A, 371, 205

Soker, N. 2000, MNRAS, 312, 217

Steffen, M., \& Schönberner, D. 2000, A\&A, 357, 180 